\title{
Brevilin A promotes oxidative stress and induces mitochondrial apoptosis in U87 glioblastoma cells
}

This article was published in the following Dove Press journal:

OncoTargets and Therapy

\author{
Jie Wang ${ }^{1,2}$ \\ Miao $\mathrm{Li}^{2}$ \\ Xiaolin Cui' \\ Dongyue Lv' \\ Lingling Jin' \\ Muhammad $\mathrm{Khan}^{3}$ \\ Tonghui $\mathrm{Ma}^{\prime}$ \\ 'College of Basic Medical Sciences, \\ Dalian Medical University, Dalian, \\ China; ${ }^{2}$ Department of Neurology, \\ The China-Japan Union Hospital of \\ Jilin University, Changchun, China; \\ ${ }^{3}$ Department of Zoology, University \\ of the Punjab, Quaid-e-Azam Campus, \\ Lahore, Pakistan
}

Correspondence: Tonghui Ma College of Basic Medical Sciences, Dalian Medical University, 9 Western Section, Lvshun South Road Dalian, I16044, China Tel +864 II 86II 0278 Fax $+8641 I 86 I I 0378$

Email tonghuima@dlmedu.edu.cn

\begin{abstract}
Background: Sesquiterpene lactones are plant-derived, natural, bioactive molecules often used against inflammatory diseases in traditional Chinese medicines. Recently, sesquiterpene lactones have been reported to exhibit potent anticancer activity. In the present study, we have investigated the anticancer activity of Brevilin A, a sesquiterpene lactone component of Centipeda minima, against U87 glioblastoma cells.

Materials and methods: The cell proliferation was determined by MTT assay. Cell morphological changes were observed by phase-contrast microscopy. Flow cytometry was used to measure apoptosis. Glutathione (GSH), ROS generation, and mitochondrial membrane potential were measured using commercially available kits. The expression of proteins was measured by Western blotting analysis.

Results: Brevilin A inhibited the proliferation of, and induced severe morphological changes and apoptotic cell death in, U87 glioblastoma cells in a dose-dependent manner. Further mechanistic study revealed that Brevilin A induces oxidative stress, as evident from ROS generation, GSH depletion, and increased phosphorylation of stress-activated proteins $\mathrm{p} 38$ and JNK. Furthermore, Brevilin A bcl-xl/bak ratio, decreased mitochondrial membrane potential and induced cytochrome c release from mitochondria into cytosol in a dose-dependent manner. Finally, Brevilin A decreased the expression of Xiap and increased the expression of cleaved forms of caspase- 9 and -3 and PARP in a dose-dependent manner.

Conclusion: Collective findings demonstrated that Brevilin A is a potent, anticancer, bioactive molecule and it effectively induces apoptosis in U87 glioblastoma cells, which is associated with induction of oxidative stress and mitochondrial dysfunction.
\end{abstract}

Keywords: Brevilin A, glioblastoma, chemotherapy, apoptosis, oxidative stress, mitochondrial dysfunction

\section{Introduction}

Glioblastoma is the most common and aggressive primary brain cancer and accounts for more than $60 \%$ of malignant gliomas. ${ }^{1}$ At present, glioblastoma is treated with surgery followed by radiotherapy and chemotherapy. Currently, temozolomide and bevacizumab are the only Food and Drug Administration (US FDA)-approved gold standard chemotherapeutic drugs used to treat newly diagnosed glioblastoma and recurrent glioblastoma, respectively. Despite recent advances in surgery, radiation therapy, and chemotherapy, the prognosis of glioblastoma remains very poor with median survival of approximately $13-16$ months. ${ }^{2}$ Therefore, there is an ongoing need for the identification of novel bioactive molecules and their underlying mechanisms to improve the outcome of chemotherapy.

Sesquiterpene lactones are plant-derived, natural, bioactive molecules often used against inflammation and cancer in traditional Chinese medicine. ${ }^{3}$ In recent years, 
increasing attention has been paid to investigate the anticancer mechanism of sesquiterpene lactones against various human cancers. ${ }^{4-6}$ Brevilin $\mathrm{A}$ is a sesquiterpene lactone component of Centipeda minima - a traditional Chinese medicinal herb used to treat asthma and cough. ${ }^{7}$ The extracts of Centipeda minima have been reported to exhibit antibacterial, antiproliferative, antioxidant, anti-inflammatory, and antitumor activities. ${ }^{8}$ Brevilin A has been shown to inhibit proliferation of various human cancer cells including A549, HepG2, HeLa, A875, and CT26 cells. ${ }^{7}$ In the present study, we have investigated the anticancer mechanism of Brevilin A in U87 glioblastoma cells. Our data demonstrated that Brevilin A inhibits growth and induces mitochondrial apoptosis in U87 glioblastoma cells.

\section{Materials and methods}

\section{Antibodies and reagents}

Brevilin A (Figure 1A) was purchased from Dalian Meilun Biotechnology, Co., Ltd., and its purity was more than $98 \%$ as determined by HPLC. Temozolomide was obtained from Selleckchem (Munich, Germany). DMEM and FBS were obtained from Gibco (Eggenstein, Germany). Penicillin and streptomycin were purchased from Solarbio Co., Ltd. (Beijing, China). Annexin V-FITC/PI apoptosis detection kit, Reactive Oxygen Species (ROS) assay kit, mitochondrial membrane potential (MMP) assay kit with JC-1, MTT reagent, Western blotting reagents, and dimethyl sulfoxide (DMSO) were purchased from Beyotime Institute of Biotechnology (Haimen, Jiangsu, China). The glutathione (GSH) assay kit was obtained
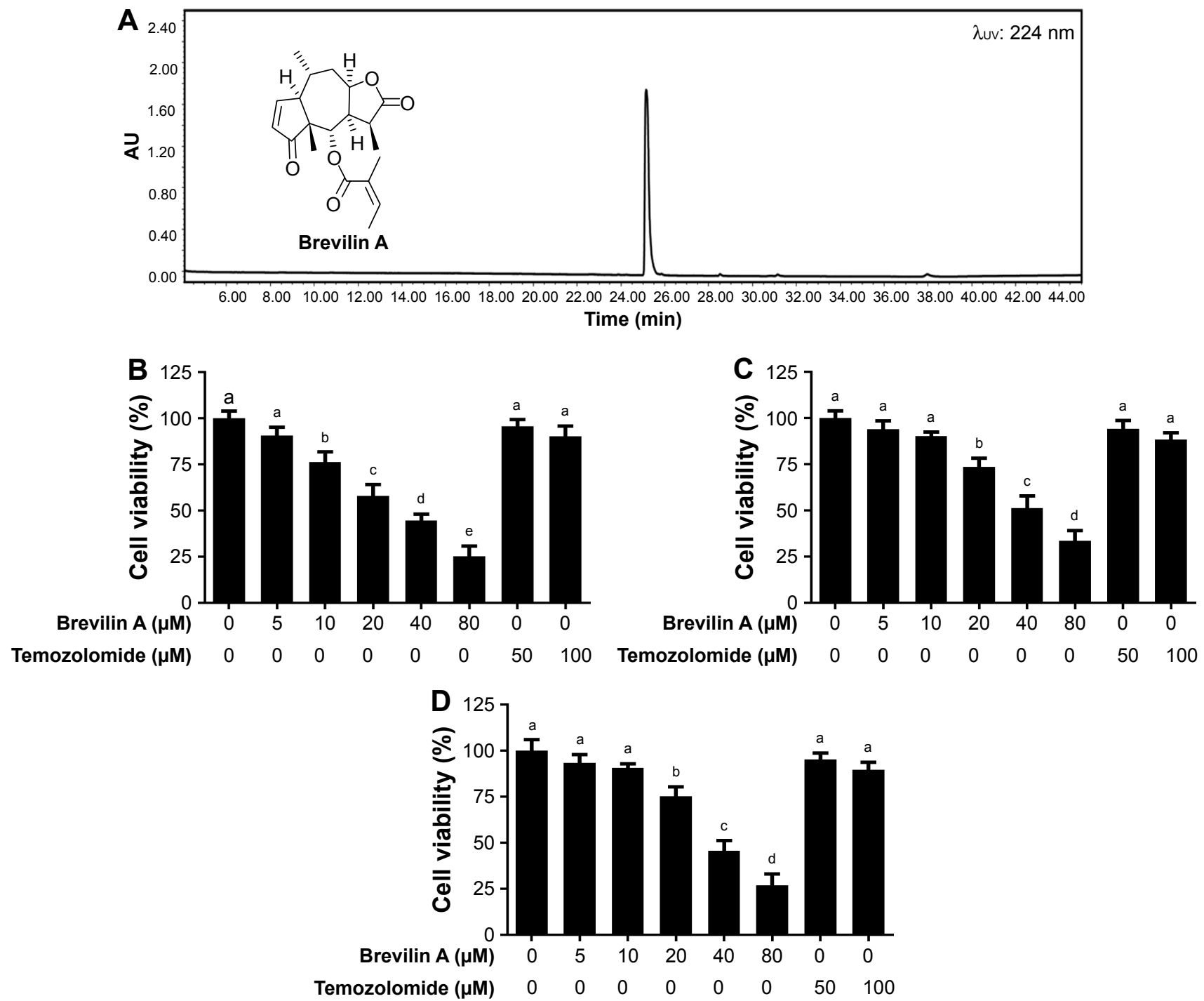

Figure I Brevilin A decreased cell viability of glioblastoma cells.

Notes: (A) Chemical structure and HPLC purity peak of Brevilin A. (B) U87, (C) U373, and (D) LN229 glioblastoma cells were treated with Brevilin A in a dose-dependent manner for 24 hours. Temozolomide was used as the positive control. The cell viability was determined as described in Materials and methods. Data are expressed as mean \pm SEM of three independent experiments. Columns with different superscript letters showed statistically significant differences $(P<0.05)$. 
from Nanjing Jiancheng Bio-Engineering Institute (Nanjing, Jiancheng, China). The primary antibodies for cleaved forms of caspase-9, caspase-3, and PARP as well as p-JNK, JNK p-p38, and p38 were obtained from Cell Signaling Technology (Beverly, MA, USA) whereas primary antibodies for Bax, Bak, Bcl-2, Bcl-xL, Xiap, Cytochrome c, and GAPDH were obtained from Proteintech (Wuhan, China). Horseradish peroxidase (HRP)-conjugated secondary antibodies were purchased from Sigma-Aldrich (St Louis, MO, USA).

\section{Cell culture and treatment}

Human U87, U373, and LN229 glioblastoma cells were obtained from the American Type Culture Collection (ATCC). The cells were cultured in DMEM supplemented with $10 \%$ FBS, 100 units $/ \mathrm{mL}$ penicillin, and $100 \mu \mathrm{g} / \mathrm{mL}$ streptomycin at $37^{\circ} \mathrm{C}$ with $5 \% \mathrm{CO}_{2}$ in a humidified atmosphere. Brevilin A was dissolved in DMSO to obtain a 5-mM stock solution and kept at $4{ }^{\circ} \mathrm{C}$ in the dark, protected from light. Cells were treated with Brevilin A dissolved in DMSO, with a final DMSO concentration of $0.5 \%$ which was found to be non-toxic as determined by our pilot experiment. Control cells were treated with $0.5 \%$ DMSO.

\section{Determination of cell viability by MTT assay}

U87, U373, and LN229 glioblastoma cells were cultured in 96-well plates in triplicate overnight. Cells were treated with Brevilin A and temozolomide as indicated for 24 hours at $37^{\circ} \mathrm{C}$ in $\mathrm{CO}_{2}$ incubator. Following Brevilin A treatment, $10 \mu \mathrm{L}$ MTT reagent $(5 \mathrm{mg} / \mathrm{mL}$ ) was added to each well and cells were further incubated at $37^{\circ} \mathrm{C}$ for 4 hours in the dark. Subsequently, the medium was replaced and $150 \mu \mathrm{L}$ DMSO was added to dissolve formazan crystals. After shaking the plate for 5 minutes, absorbance was measured at $570 \mathrm{~nm}$ on a Synergy new HTS multimode microplate reader (BioTek). The percentage of cell viability was calculated as described previously. ${ }^{9}$

\section{Observation of cell morphological changes}

U87, U373, and LN229 glioblastoma cells were seeded into six-well plates in triplicate and incubated at $37^{\circ} \mathrm{C}$ in an incubator overnight. The cells were exposed to 0,10 , and $20 \mu \mathrm{M}$ Brevilin A for 24 hours. After the Brevilin A treatment, the cell morphological changes were observed under a phasecontrast microscope (DMIL LED, Leica) and photographed using a DFC450C camera (Leica).

\section{Apoptosis assay}

An apoptosis assay was conducted using Annexin V/PI double-staining kit (Beyotime, Haimen, Jiangsu, China). Briefly, U87 glioblastoma cells were cultured in six-well plates at $37^{\circ} \mathrm{C}$ in an incubator overnight. The cells were treated with 10 and $20 \mu \mathrm{M}$ Brevilin A for 24 hours. Following drug treatment, adherent and floating cells were collected, washed with PBS, and re-suspended in binding buffer. The samples were incubated with $5 \mu \mathrm{L}$ Annexin $\mathrm{V}$ for 10 minutes in the dark, followed by incubation with $10 \mu \mathrm{L}$ PI for another 15 minutes in the dark at room temperature according to the manufacturer's instructions for the kit. After filtration, the cell samples were analyzed by flow cytometry (BD Accuri $\mathrm{C}^{6}$ ) for the detection of apoptotic cells.

\section{Determination of ROS generation}

Intracellular ROS generation was detected using a dichlorodihydro-fluorescein diacetate (DCFH-DA) ROS assay kit (Beyotime, Haimen, Jiangsu, China). Briefly, U87 glioblastoma cells were cultured in a six-well plate in triplicates and treated with 10 and $20 \mu \mathrm{M}$ Brevilin A for 4 hours. Following Brevilin A treatment, the cells were washed with PBS and incubated with DCFH-DA for 30 minutes in the dark according to the manufacturer's instructions. The cells were washed with DMEM three times to remove extracellular DCFH-DA. The samples were analyzed for dichlorofluorescein (DCF) fluorescence at an excitation wavelength (ex.) of $488 \mathrm{~nm}$ and an emission wavelength (em.) of $525 \mathrm{~nm}$ by a fluorescence microplate reader (Synergy Neo HTS Multi-Mode Microplate Reader, BioTek).

\section{Measurement of intracellular GSH}

The intracellular GSH was measured using a glutathione reduced assay kit (Nanjing, Jiancheng, China). The cells were cultured in six-well plates in triplicates and treated with 10 and $20 \mu \mathrm{M}$ Brevilin A for 4 hours. The cells were collected, washed with PBS, and samples were prepared in accordance with the manufacturer's instructions. Finally, the intracellular GSH was measured using a fluorescent spectrophotometer (Synergy Neo HTS Multi-Mode Microplate Reader, BioTek) at a wavelength of $405 \mathrm{~nm}$. The GSH contents were expressed as nmol GSH/mg protein.

\section{Determination of mitochondrial membrane potential}

The mitochondrial membrane potential was measured using a JC-1 probe according to the manufacturer's instructions 
(Beyotime). Briefly, the cells were cultured in six-well plates and treated with 10 and $20 \mu \mathrm{M}$ Brevilin A for 24 hours. After drug treatment, the cells were harvested, washed with serum-free DMEM, and incubated with JC-1 fluorescent probe for 20 minutes in the dark at $37^{\circ} \mathrm{C}$. Following incubation, the cells were washed and the fluorescence distribution of JC-1 monomers (green fluorescence; ex. $\lambda 490 \mathrm{~nm}$ and em. $\lambda 530 \mathrm{~nm}$ ) and J-aggregates (red fluorescence; ex. $\lambda$ $525 \mathrm{~nm}$ and em. $\lambda 590 \mathrm{~nm}$ ) was measured by a fluorescence microplate reader (Synergy Neo HTS Multi-Mode Microplate Reader, BioTek). The MMP was calculated by a decrease in $\mathrm{red} /$ green fluorescence-intensity ratio.

\section{Immunoblotting}

U87 glioblastoma cells were cultured in six-well plates and exposed to 10 and $20 \mu \mathrm{M}$ Brevilin A for the indicated time points. The floating and adherent cells were collected and total cell lysates were prepared as we have described previously. ${ }^{10}$ Cytosolic fractions were extracted using a mitochondrial/cytosolic fractionation kit (BioVision). A BCA protein assay kit was used to measure protein concentration (Beyotime Biotechnology). A total of $30 \mu \mathrm{g}$ protein was electrophoresed on 10\%-12\% SDS-PAGE and transferred to a polyvinylidene difluoride membrane. After blocking with 5\% skimmed milk, the membranes were washed with Tris-buffered saline-Tween (TBST). The membranes were incubated with p-JNK $(1: 1,000)$, JNK $(1: 1,000)$, p-p38 (1:1,000), p38 (1:1,000), bcl-2 (1:500), bcl-xL (1:500), bax (1:500), bak $(1: 500)$, cleaved caspase-9 $(1: 1,000)$, cleaved caspase-3 $(1: 1,000)$, cytochrome c $(1: 1,000)$, and GAPDH $(1: 1,000)$ overnight at $4^{\circ} \mathrm{C}$. After washing with TBST solution, the membranes were incubated with respective HRP-conjugated secondary antibodies for 1 hour at room temperature. Finally, the membranes were washed and the immunoreactive bands were detected by Immobilon Western chemiluminescent HRP substrate (Millipore, Billerica, MA, USA) using a MicroChemi 4.2 imaging system (DNR Bio-Imaging system). All the blots shown are representative of three separate experiments. GAPDH was used as the loading control.

\section{Statistical analysis}

Data are expressed as mean \pm standard error mean (SEM) of three independent experiments and statistically compared with the control group or within the groups using one-way ANOVA followed by Tukey's Multiple Comparison Test; $P<0.05$ was considered indicative of statistical significance.

\section{Results \\ Brevilin A inhibits proliferation and induces cytotoxicity in U87 glioblastoma cells}

The anti-proliferative effect of Brevilin A on glioblastoma was assessed using U87, U373, and LN229 cells. To observe the anti-proliferative effect of Brevilin A, we conducted an MTT assay. The data demonstrated that Brevilin A inhibited the cell proliferation of all three cell lines in a dose-dependent manner (Figure 1). The $\mathrm{IC}_{50}$ values of Brevilin A against U87, LN229, and U373 were found to be approximately 30, 36, and $40 \mu \mathrm{M}$, respectively, at the 24 -hour time point. Despite lower concentrations of Brevilin A, the anti-proliferative effect of Brevilin A on all three glioma cell lines was significantly higher when compared to temozolomide. Temozolomide, even at higher concentrations $(50$ and $100 \mu \mathrm{M})$, failed to inhibit proliferation significantly $(P<0.05)$. Because U87 cells were found to be most sensitive to Brevilin A, we selected U87 cells for further mechanistic study. Moreover, less toxic concentrations ( 10 and $20 \mu \mathrm{M}$ ) of Brevilin A were selected to investigate the anticancer mechanism. To observe the effect of Brevilin A on cell morphology, we exposed the cells to 10 and $20 \mu \mathrm{M}$ Brevilin A for 24 hours. Following drug treatment, the cell morphological changes were observed microscopically. As shown in Figure 2A, Brevilin A induced severe morphological changes in U87 glioma cells in a dose-dependent manner. These morphological alterations were associated with markers of cell death including rounding of cells, loss of anchorage from culture plate, and decreased total number of cells. The data demonstrated that Brevilin A decreased cell viability and induced cell death in glioblastoma cells.

\section{Brevilin A induces apoptotic cell death in U87 glioblastoma cells}

Because Brevilin A induced cytotoxicity in U87 glioblastoma cells, we were interested to know the nature of cell death. For this, we conducted flow cytometric analysis of apoptosis using an Annexin V-FITC and PI double-staining kit. The cells residing in the Q1-LL region are both Annexin V-FITC- and PI-negative cells. The cells present in the Q1-LR region were Annexin V positive whereas cells found in the Q1-UR region were Annexin V-PI-positive cells. Annexin V-positive cells are considered as early apoptotic cells whereas Annexin V-PI-positive cells are considered as late apoptotic or necrotic cells. The Q1-UL region is for PI-stained DNA debris. The results showed that Brevilin A induces apoptotic cell death in U87 glioblastoma cells 
A

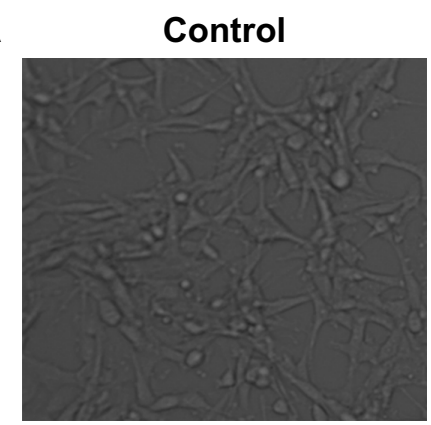

B

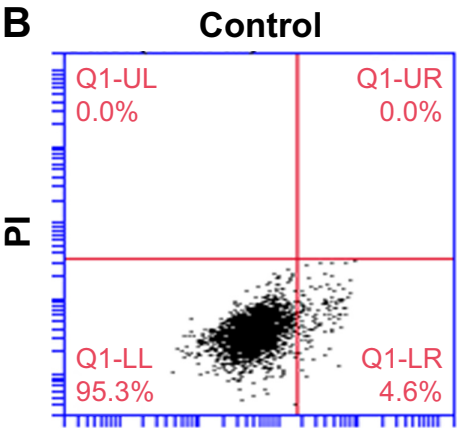

Annexin V-FITC

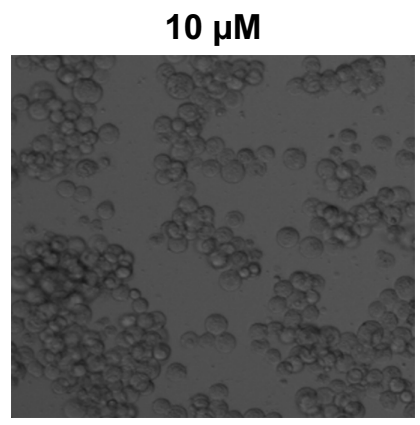

$10 \mu \mathrm{M}$

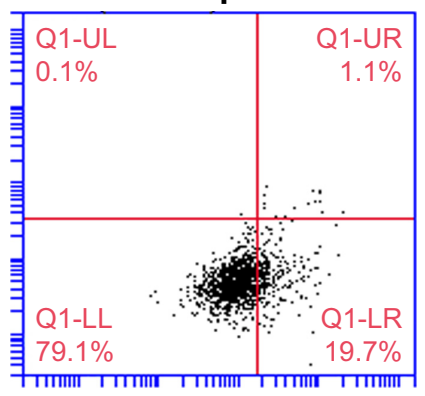

Annexin V-FITC
$20 \mu \mathrm{M}$

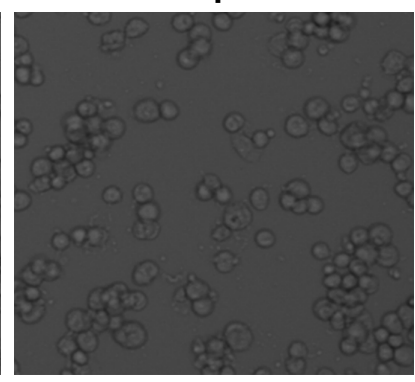

$20 \mu \mathrm{M}$

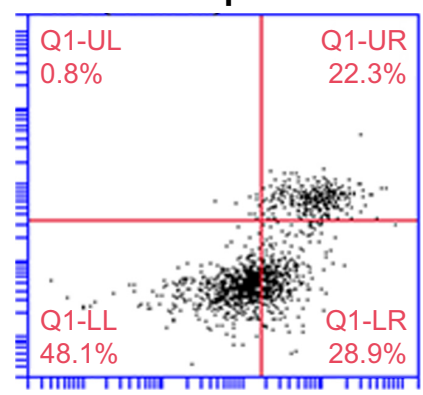

Annexin V-FITC

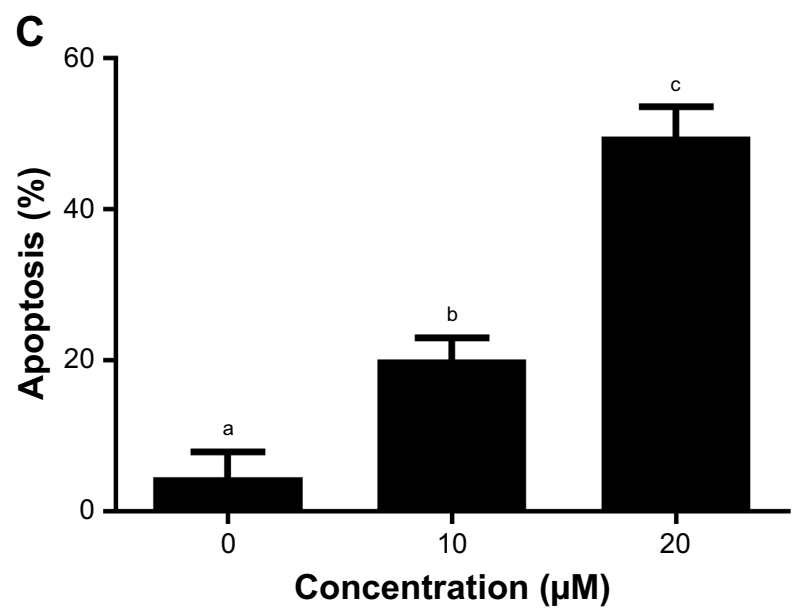

Figure 2 Brevilin A induces morphological changes and apoptosis in U87 glioblastoma cells.

Notes: (A) U87 cells were cultured in 96-well plates overnight and treated with indicated concentrations of Brevilin A for 24 hours. Following treatment, the cells, morphological changes were observed and photographed. Scale bar $=100 \mu \mathrm{m}$. (B) U87 cells were cultured in six-well plates overnight and then treated with indicated concentrations of Brevilin A for 24 hours. The floating and adherent cells were collected, washed with PBS, and incubated with Annexin V-FITC and PI for I5 minutes in the dark according to the manufacturer's instructions. After filtration, the samples were analyzed by flow cytometry for the percentage of apoptotic cells. (C) Data are expressed as mean \pm SEM of three independent experiments. Columns with different superscript letters differ significantly $(P<0.05)$.

in a dose-dependent manner (Figure 2B and C). Moreover, Brevilin A induces early apoptosis at $10 \mu \mathrm{M}$ concentration whereas, at $20 \mu \mathrm{M}$, it induces both early as well as late apoptosis in U87 glioblastoma cells.

\section{Brevilin A induces oxidative stress in U87 glioblastoma cells}

In normal cells, a precise balance exists between ROS generation and the antioxidant system of the cells. ROS are produced in cells as a result of the metabolic activities of cells, which are effectively neutralized by the scavenging ability of the antioxidant system of cells. However, disruption of this balance, such as by overproduction of ROS and downregulation of the antioxidant system of the cells, leads to oxidative stress. ${ }^{11}$ Sesquiterpene lactones have been shown to induce oxidative stress in various cancer cells; ${ }^{4,12,13}$ therefore, we were interested to know whether Brevilin A could induce oxidative stress in U87 glioblastoma cells. First, we determined intracellular ROS generation by staining the cells with DCFH-DA, which is freely permeable across the 
plasma membrane. It is converted into DCFH by the activity of the intracellular esterases. DCFH is impermeable across the plasma membrane and, inside the cells, it is oxidized into fluorescent-probe DCF by ROS. Therefore, the fluorescence intensity of DCF inside the cells is directly proportional to intracellular ROS generation. ${ }^{13}$ The data demonstrated that Brevilin A treatment increased ROS generation in U87 glioblastoma cells (Figure 3A). Next, we measured the intracellular reduced GSH level in U87 glioblastoma cells in response to Brevilin A treatment by using a commercially available kit. The data indicated that Brevilin A significantly $(P<0.05)$ decreased intracellular reduced GSH as shown in Figure 3B.

\section{Brevilin A induces JNK and p38 activation} JNK and p38 are members of MAPK family proteins and are activated in response to various cellular stresses including oxidative stress. ${ }^{10}$ Brevilin A induced oxidative stress in U87 glioblastoma cells in the present study; therefore, we evaluated whether Brevilin A could induce activation of the stress-activated proteins JNK and p38. For this, we exposed the cells to Brevilin A for 4 hours and checked activation of JNK and p38. The results showed that Brevilin A increased the phosphorylation of JNK and p38 in a dose-dependent manner without affecting the expression of JNK and p38 (Figure 3C).

\section{Brevilin A induces mitochondrial apoptosis in U87 glioblastoma cells}

To get better insight into Brevilin A-induced apoptosis in U87 glioblastoma cells, we measured the expression of bcl-2 family proteins by Western blotting analysis. The data demonstrated that Brevilin A decreased the expression of antiapoptotic $\mathrm{Bcl}-\mathrm{xL}$ protein whereas it increased the expression
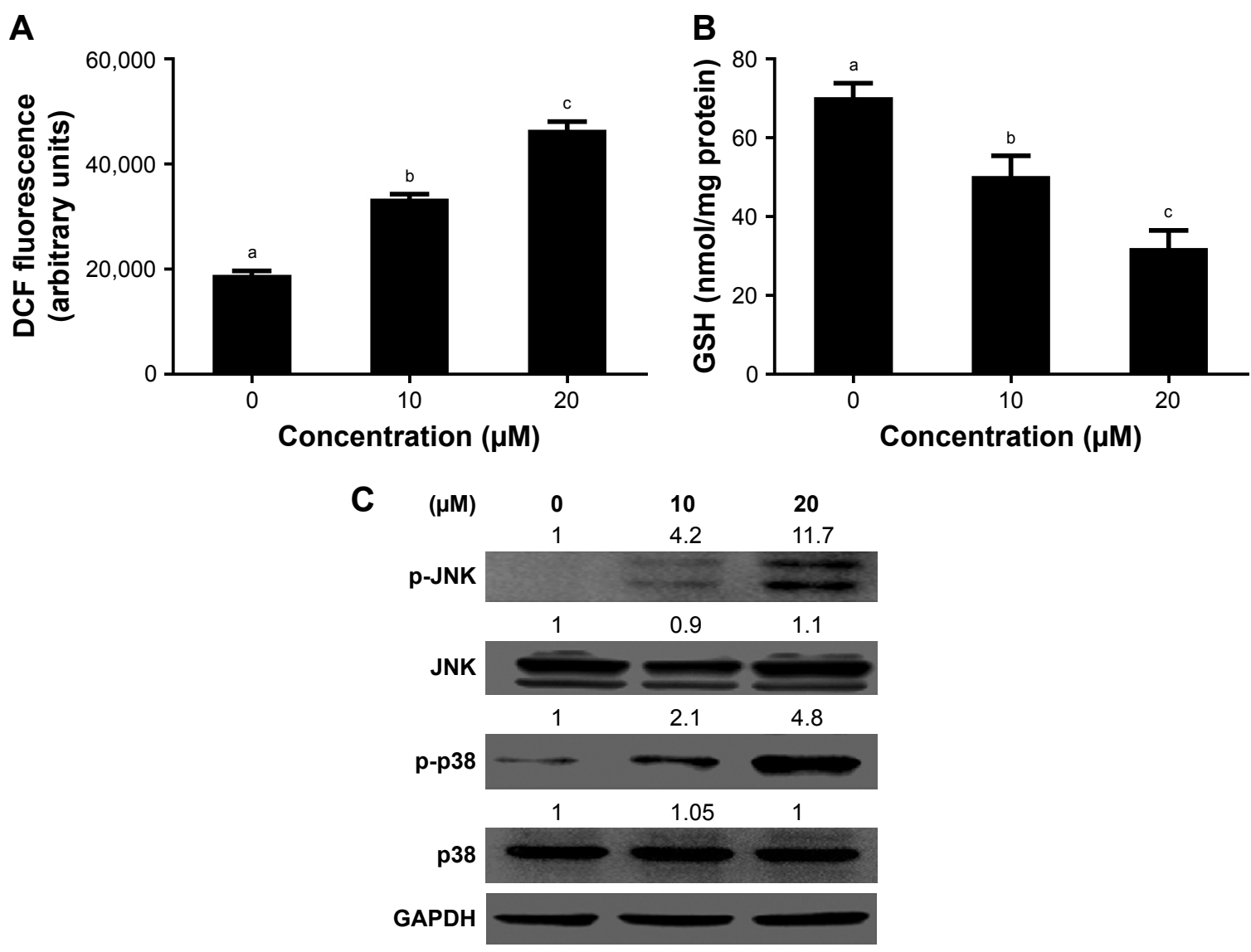

Figure 3 Brevilin A promotes oxidative stress in U87 glioblastoma cells.

Notes: (A) The cells were treated with 0, 10, and $20 \mu \mathrm{M}$ Brevilin A for 4 hours, and ROS generation was measured by staining the cells with dichloro-dihydro-fluorescein diacetate (DCFH-DA) according the manufacturer's instructions. (B) The cells were treated with indicated doses of Brevilin A for 4 hours, and intracellular GSH was determined according to the manufacturer's instructions. Values are expressed as nmol/mg of proteins. Data (A \& B) are expressed as mean \pm SEM of three different experiments. Columns with different superscript letters differ significantly $(P<0.05)$. (C) The cells were cultured in six-well plates and treated with indicated concentrations of Brevilin A for 4 hours. The cells were harvested, washed with cold PBS, and cell lysates were prepared and subjected to Western blots for the expression of P-JNK, JNK, $\mathrm{P}-\mathrm{p} 38$, and $\mathrm{p} 38$. GAPDH was used as the loading control. The numerical values above the bands represent the relative density of the bands normalized to total protein ( $\mathrm{p}-\mathrm{JNK}$ / JNK and p-p38/p38) from three different experiments.

Abbreviations: DCF, dichlorofluoresceinl; GSH, glutathione. 
of pro-apoptotic Bak protein. However, no change in the expression of anti-apoptotic $\mathrm{Bcl}-2$ protein and pro-apoptotic Bax protein was observed (Figure 4A). To support our data, we further measured the expression of cytochrome $\mathrm{c}$ in cytosolic fractions. The data showed that Brevilin A treatment induced release of cytochrome $\mathrm{c}$ from the mitochondria into the cytosol (Figure 4A). Next, we determined mitochondrial membrane potential in U87 glioblastoma cells by using the JC-1 fluorescent probe. In healthy cells, JC-1 forms complexes known as J-aggregates, which show intense red fluorescence; however, in cells with disrupted MMP, JC-1 remains in monomeric form and manifests green fluorescence. Thus, MMP is calculated by a decrease in red/green fluorescence ratio. The data demonstrated that Brevilin A decreased red/green fluorescence ratio (MMP) in U87 glioblastoma cells in a dose-dependent manner (Figure 4B). These findings clearly indicate that Brevilin A induces at least, in part, mitochondrial apoptosis in U87 glioblastoma cells.

A
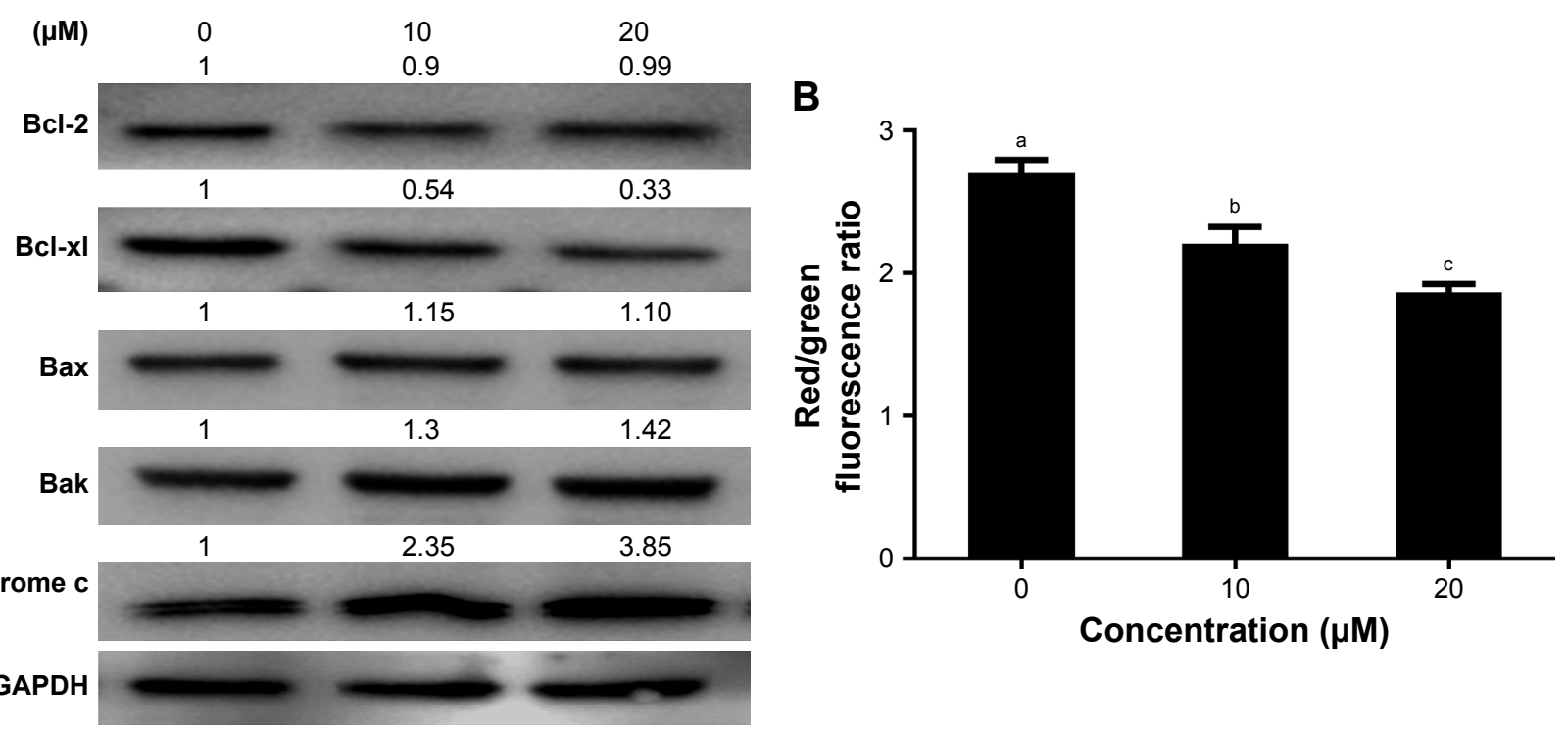

Cytochrome c

GAPDH

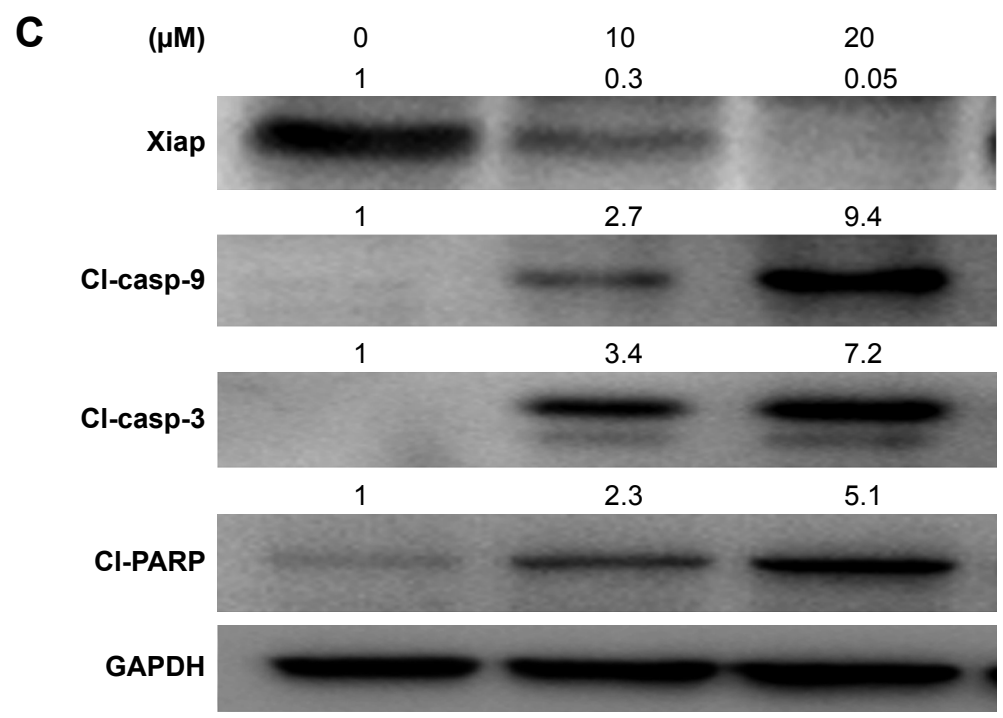

Figure 4 Brevilin A induces intrinsic apoptosis in U87 glioblastoma cells.

Notes: (A) U87 glioblastoma cells were treated with Brevilin A for 24 hours and cellular extracts were subjected to Western blotting analysis for the expression of Bcl-2, $\mathrm{Bcl}-\mathrm{xl}$, Bax, and Bak proteins. The expression of cytochrome $\mathrm{c}$ was determined in cytosolic extractions as described in Materials and methods. The digital values above the immunoblots show the relative density of the bands normalized to GAPDH from three different experiments. (B) U87 cells were treated with Brevilin A, and the mitochondrial membrane potential was determined using the JC-I kit. The MMP was calculated by measuring the red/green fluorescence intensity ratio. Columns with different superscript letters differ significantly $(P<0.05)$. (C) The cells were incubated with indicated concentrations of Brevilin $A$ for 24 hours. Following drug treatment, the total cell lysates were prepared and subjected to Western blotting analysis for the expression of cleaved forms of caspase-9, caspase-3, and PARP. GAPDH was used as loading control. The values above the bands show the relative density of the bands normalized to GAPDH from three repeated experiments. 


\section{Brevilin A inhibits xiap expression and induces caspase activation and PARP cleavage}

As a result of mitochondrial dysfunction, several pro-apoptotic molecules including cytochrome $\mathrm{c}$ are released from the mitochondria and activate the caspase-cascade. The activation of caspases and cleavage of PARP are specific markers of apoptotic cell death. ${ }^{15}$ Caspases are activated by cleavage. Therefore, we measured the expression of cleaved forms of caspase-9 and -3 as well as PARP in control and Brevilin A-treated U87 glioblastoma cells. As shown in Figure 4C, Brevilin A increased the expression of cleaved forms of caspase-9, caspase-3, and PARP in a dose-dependent manner. Moreover, we measured the expression of the X-linked inhibitor of apoptosis (xiap), and data demonstrated that Brevilin A inhibited the expression of xiap in a dose-dependent manner (Figure 4C). The collective data demonstrate that Brevilin A induces apoptotic cell death in U87 glioblastoma cells.

\section{Discussion}

Glioblastoma multiforme (GBM) is the most common and aggressive primary brain tumor that develops from starshaped glial cells and accounts for at least $60 \%$ of malignant gliomas. ${ }^{1}$ Despite concerted effort to improve treatment modalities, the prognosis of GBM remains very poor. ${ }^{2}$ Identification of novel bioactive molecules and investigation of their anti-glioma mechanism is, therefore, necessary to improve the outcomes of chemotherapy. Sesquiterpene lactones are plant-derived, natural, bioactive entities that have a long history of use against inflammatory diseases in traditional Chinese medicine. ${ }^{3}$ In the last few decades, sesquiterpene lactones have drawn the attention of scientists and pharmacists due to promising anticancer activity against various human malignancies. In the present study, we have identified and investigated the anti-glioma activity of Brevilin A - a sesquiterpene lactone component of Centipeda minima.

Sesquiterpene lactone compounds are emerging as a promising alternative to existing chemotherapy. The anticancer activity of sesquiterpene lactones has been believed to be mediated by the alkylating centers. The typical alkylating centers found in sesquiterpene lactone compounds are $\alpha$-methylene- $\gamma$-lactone, $\alpha-\beta$ cyclopentenone, unsaturated esters in side chains, and epoxides. ${ }^{3,16}$ The alkylating centers of sesquiterpene lactones react through MichaelType addition with biological nucleophiles such as thiolcontaining cysteine residues in proteins, thereby making stable adducts. As sesquiterpene lactones could conjugate with a large number of cysteine-containing proteins, they can modulate the expressions of multiple target molecules. ${ }^{3}$ Brevilin A - a sesquiterpene lactone compound which lacks a typical $\alpha$-methylene- $\gamma$-lactone moiety but possesses a cyclopentenone alkylating center - has been shown to inhibit proliferation in various cancer cells including A549, HepG2, HeLa, A875, and CT26 cells. ${ }^{7}$ In line with a previous report, Brevilin A effectively inhibited proliferation and induced apoptotic cell death in U87 glioblastoma cells in the present study. ${ }^{?}$

It is well established now that cancer cells survive under higher oxidative stress as compared to normal cells. This adaptive feature of cancer cells is implicated in various cellular processes including cell survival, proliferation, angiogenesis, and metastasis. ${ }^{14,15}$ Modern research has shown that this adaptation of cancer cells could be exploited for therapeutic benefits. ${ }^{4}$ As cancer cells contain higher ROS level, exposing cancer cells to ROS-inducing phytochemical could enhance the ROS level above the toxic threshold. Compared to normal cells, in cancer cells, the toxic threshold can be easily achieved by exposing them to exogenous ROS-inducing phytochemicals. Indeed, induction of cell death in cancer cells by exposing them to an ROS-inducing phytochemical has been shown to be feasible in various in vitro cell culture studies and in vivo animal models. ${ }^{17-19}$ Unlike prevailing chemotherapeutics, an ROS-based drug development strategy holds the promise to kill cancer cells of multiple origins more effectively, irrespective of their genotype and heterogeneity. ${ }^{4}$ Among various classes of bioactive phytochemicals, sesquiterpene lactones have been reported to be the most potent ROS inducers. The $\alpha$-methylene- $\gamma$ lactone structures of sesquiterpene lactone compounds readily react with reduced GSH and thereby disrupt the redox balance of the cells; this ultimately results in mitochondrial dysfunction and induction of apoptosis. ${ }^{4,20-22}$ Other research reports showed that the cyclopentenone moiety reacts more readily with GSH than the $\alpha$-methylene- $\gamma$-lactone moiety. ${ }^{23}$ Brevilin A does not contain a typical $\alpha$-methylene- $\gamma$-lactone structure but possesses a cyclopentenone structure; therefore, we were interested to know whether Brevilin A could induce oxidative stress in U87 glioblastoma cells. We found that Brevilin A effectively decreased the GSH level and provoked ROS generation in U87 glioblastoma cells in a dose-dependent manner at an early time point (4 hours). These findings are further supported by a previous study by You et al, who showed that Brevilin A increases ROS 
generation in C26 colon adenocarcinoma cells. ${ }^{7}$ Similarly, dihydrohelenalin - which lacks $\alpha$-methylene- $\gamma$-lactone but contains cyclopentenone - could effectively react with GSH. ${ }^{16}$ Although Brevilin A induced oxidative stress and apoptosis in cancer cells, its effect on normal cells remains to be determined.

JNK and p38 are members of the MAPK family of proteins, which are activated in response to various cellular stresses including oxidative stress. ${ }^{10}$ Because Brevilin A induced oxidative stress in U87 glioblastoma cells, we assessed whether Brevilin A could activate the stressactivated JNK and p38 MAPK proteins. We found that Brevilin A remarkably increased the phosphorylation of stress-activated JNK and p38 proteins, further supporting the hypothesis that Brevilin A induces oxidative stress in U87 glioblastoma cells. The data are in line with previous reports which showed that isoalantolactone - a sesquiterpene lactone - induces p38 and JNK activation in breast cancer cells. $^{24,25}$

Mitochondria are very important components of the apoptotic machinery and play an important role in the induction of intrinsic apoptosis. Mitochondrial apoptosis is initiated by the modulation of $\mathrm{Bcl}-2$ family proteins, which results in the opening of the mitochondrial permeability transition pore, disruption of MMP, and subsequent release of cytochrome c from the mitochondrial intermembrane space into the cytosol that ultimately binds with and activates caspase-9. Activated caspase-9 then activates caspase-3, which is the main executioner of apoptosis. Ultimately, activated caspase- 3 cleaves PARP and thereby induces apoptosis. The activation of caspase- 3 and cleavage of PARP are the characteristic features of apoptotic cell death. ${ }^{15}$ Previous reports demonstrated that sesquiterpene lactone compounds effectively induce mitochondrial apoptosis in various human cancer cells. .,11,20,26,27 $^{-1}$ Here, in this study, we found that Brevilin A modulated the expression of Bcl-2 family proteins, decreased MMP, and induced cytochrome $\mathrm{c}$ release from the mitochondria into the cytosol. Furthermore, we showed that Brevilin A treatment remarkably increased the expressions of cleaved forms of caspase-9, caspase-3, and PARP and inhibited the expression of Xiap in U87 glioblastoma cells. Our data are further supported by a recent research report in which Brevilin A has been shown to disrupt the modulation of MMP and Bcl-2 family proteins in CT2 6 cells. ${ }^{7}$ Although the induction of oxidative stress, modulation of Bcl-2 family proteins, disruption of MMP, and release of cytochrome $\mathrm{c}$ from the mitochondria into the cytosol are considered the characteristic features of mitochondrial apoptosis, further studies are warranted to explore the exact molecular mechanism of Brevilin Ainduced mitochondrial apoptosis in glioblastoma cells.

\section{Conclusion}

In conclusion, our data demonstrated that Brevilin A disrupted the redox balance by decreasing the intracellular reduced GSH and increasing ROS generation in U87 glioblastoma cells. Moreover, Brevilin A activated the JNK and p38 MAPK proteins. The induction of apoptosis was found to be associated with increased $\mathrm{Bak} / \mathrm{Bcl}-\mathrm{xL}$ ratio, MMP dissipation, cytochrome c release, activation of caspases (9 and 3), cleavage of PARP, and inhibition of Xiap expression. Our findings suggest that Brevilin A may become a promising anti-glioma agent. However, further study is needed to determine Brevilin A penetration through the blood-brain barrier (BBB) and validate its in vitro anticancer activity in animal models, which are the major limitations of this study.

\section{Acknowledgments}

This study is supported by the National Natural Science Youth Fund of the People's Republic of China (grant no 81601070), the National Natural Science Fund of the People's Republic of China (grant no 81771304), and the Health Special Fund of Jilin Province, China (2016-2018).

\section{Author contributions}

Muhammad Khan and Tonghui Ma designed the research project. Jie Wang, Miao Li, Xiaolin Cui, Dongyue Lv, and Lingling Jin conducted the experiments. Data were analyzed by Muhammad Khan and Tonghui Ma. The manuscript was written by Jie Wang and approved by Muhammad Khan. All authors contributed to data analysis, drafting and revising the article, gave final approval of the version to be published, and agree to be accountable for all aspects of the work.

\section{Disclosure}

The authors report no conflicts of interest in this work.

\section{References}

1. Hanif F, Muzaffar K, Perveen K, Malhi SM, Simjee S. Glioblastoma Multiforme: A Review of its Epidemiology and Pathogenesis through Clinical Presentation and Treatment. Asian Pac J Cancer Prev. 2017; 18(1):3-9.

2. Lau D, Magill ST, Aghi MK. Molecularly targeted therapies for recurrent glioblastoma: current and future targets. Neurosurg Focus. 2014;37(6):E15.

3. Ghantous A, Gali-Muhtasib H, Vuorela H, Saliba NA, Darwiche N. What made sesquiterpene lactones reach cancer clinical trials? Drug Discov Today. 2010;15(15-16):668-678. 
4. Maryam A, Mehmood T, Zhang H, Li Y, Khan M, Ma T. Alantolactone induces apoptosis, promotes STAT3 glutathionylation and enhances chemosensitivity of A549 lung adenocarcinoma cells to doxorubicin via oxidative stress. Sci Rep. 2017;7(1):6242.

5. Silva Castro ED, Alvesantunes LA, Revoredo Lobo JF, et al. Antileukemic Properties of Sesquiterpene Lactones: A systematic review. Anticancer Agents Med Chem. 2018;18(3):323-334.

6. Ren Y, Yu J, Kinghorn AD. Development of Anticancer Agents from Plant-Derived Sesquiterpene Lactones. Curr Med Chem. 2016;23(23): 2397-2420.

7. You P, Wu H, Deng M, Peng J, Li F, Yang Y. Brevilin A induces apoptosis and autophagy of colon adenocarcinoma cell CT26 via mitochondrial pathway and PI3K/AKT/mTOR inactivation. Biomed Pharmacother. 2018;98:619-625.

8. Chan CO, Jin DP, Dong NP, Chen SB, Mok DK. Qualitative and quantitative analysis of chemical constituents of Centipeda minima by HPLC-QTOF-MS \& HPLC-DAD. J Pharm Biomed Anal. 2016;125: 400-407.

9. Mehmood T, Maryam A, Tian X, Khan M, Ma T. Santamarine Inhibits NF- $\kappa$ B and STAT3 Activation and Induces Apoptosis in HepG2 Liver Cancer Cells via Oxidative Stress. J Cancer. 2017;8(18):3707-3717.

10. Maryam A, Mehmood T, Yan Q, Li Y, Khan M, Ma T. Proscillaridin A Promotes Oxidative Stress and ER Stress, Inhibits STAT3 Activation, and Induces Apoptosis in A549 Lung Adenocarcinoma Cells. Oxid Med Cell Longev. 2018;2018:3853409-3853417.

11. Khan M, Yi F, Rasul A, et al. Alantolactone induces apoptosis in glioblastoma cells via GSH depletion, ROS generation, and mitochondrial dysfunction. IUBMB Life. 2012;64(9):783-794.

12. Mehmood T, Maryam A, Zhang H, Li Y, Khan M, Ma T. Deoxyelephantopin induces apoptosis in HepG2 cells via oxidative stress, NF- $\kappa$ B inhibition and mitochondrial dysfunction. Biofactors. 2017;43(1):63-72.

13. Khan M, Ding C, Rasul A, et al. Isoalantolactone induces reactive oxygen species mediated apoptosis in pancreatic carcinoma PANC-1 cells. Int J Biol Sci. 2012;8(4):533-547.

14. Khan M, Maryam A, Qazi JI, Ma T. Targeting Apoptosis and Multiple Signaling Pathways with Icariside II in Cancer Cells. Int J Biol Sci. 2015; 11(9):1100-1112.

15. Khan M, Maryam A, Zhang H, Mehmood T, Ma T. Killing cancer with platycodin D through multiple mechanisms. J Cell Mol Med. 2016; 20(3):389-402.
16. Amorim MH, Gil da Costa RM, Lopes C, Bastos MM. Sesquiterpene lactones: adverse health effects and toxicity mechanisms. Crit Rev Toxicol. 2013;43(7):559-579.

17. Asby DJ, Radigois MG, Wilson DC, et al. Triggering apoptosis in cancer cells with an analogue of cribrostatin 6 that elevates intracellular ROS. Org Biomol Chem. 2016;14(39):9322-9330.

18. Seo KH, Ryu HW, Park MJ, Mangosenone F. A Furanoxanthone from Garciana mangostana, Induces Reactive Oxygen Species-Mediated Apoptosis in Lung Cancer Cells and Decreases Xenograft Tumor Growth. Phytother Res. 2015;29(11):1753-1760.

19. Shi XJ, Yu B, Wang JW, et al. Structurally novel steroidal spirooxindole by241 potently inhibits tumor growth mainly through ROS-mediated mechanisms. Sci Rep. 2016;6:31607.

20. Khan M, Li T, Ahmad Khan MK, et al. Alantolactone induces apoptosis in HepG2 cells through GSH depletion, inhibition of STAT3 activation, and mitochondrial dysfunction. Biomed Res Int. 2013:719858.

21. Gach K, Długosz A, Janecka A. The role of oxidative stress in anticancer activity of sesquiterpene lactones. Naunyn Schmiedebergs Arch Pharmacol. 2015;388(5):477-486.

22. Gach K, Janecka A. $\alpha$-Methylene- $\gamma$-lactones as a novel class of antileukemic agents. Anticancer Agents Med Chem. 2014;14(5):688-694.

23. Schmidt TJ, Lyss G, Pahl HL, Merfort I. Helenanolide type sesquiterpene lactones. Part 5: the role of glutathione addition under physiological conditions. Bioorg Med Chem. 1999;7(12):2849-2855.

24. Li Z, Qin B, Qi X, Mao J, Wu D. Isoalantolactone induces apoptosis in human breast cancer cells via ROS-mediated mitochondrial pathway and downregulation of SIRT1. Arch Pharm Res. 2016;39(10):1441-1453.

25. Wang J, Cui L, Feng L, et al. Isoalantolactone inhibits the migration and invasion of human breast cancer MDA-MB-231 cells via suppression of the p38 MAPK/NF- $\mathrm{KB}$ signaling pathway. Oncol Rep. 2016; 36(3):1269-1276.

26. Kim SL, Trang KT, Kim SH, et al. Parthenolide suppresses tumor growth in a xenograft model of colorectal cancer cells by inducing mitochondrial dysfunction and apoptosis. Int J Oncol. 2012;41(4):1547-1553.

27. Kwak SW, Park ES, Lee CS. Parthenolide induces apoptosis by activating the mitochondrial and death receptor pathways and inhibits FAKmediated cell invasion. Mol Cell Biochem. 2014;385(1-2):133-144.
OncoTargets and Therapy

\section{Publish your work in this journal}

OncoTargets and Therapy is an international, peer-reviewed, open access journal focusing on the pathological basis of all cancers, potential targets for therapy and treatment protocols employed to improve the management of cancer patients. The journal also focuses on the impact of management programs and new therapeutic agents and protocols on

\section{Dovepress}

patient perspectives such as quality of life, adherence and satisfaction The manuscript management system is completely online and includes a very quick and fair peer-review system, which is all easy to use. Visit http://www.dovepress.com/testimonials.php to read real quotes from published authors. 